\title{
AN UNCONDITIONALLY POSITIVE AND GLOBAL STABILITY PRESERVING NSFD SCHEME FOR AN EPIDEMIC MODEL WITH VACCINATION
}

\author{
DEQIONG DING, QIANG MA, XIAOHUA DING \\ Department of Mathematics \\ Harbin Institute of Technology (Weihai), Weihai Shangdong 264209, PR China \\ e-mail: \{mathddq, mathdxh\}@163.com
}

\begin{abstract}
In this paper, a NonStandard Finite Difference (NSFD) scheme is constructed, which can be used to determine numerical solutions for an epidemic model with vaccination. Here the NSFD method is employed to derive a set of difference equations for the epidemic model with vaccination. We show that difference equations have the same dynamics as the original differential system, such as the positivity of the solutions and the stability of the equilibria, without being restricted by the time step. Our proof of global stability utilizes the method of Lyapunov functions. Numerical simulation illustrates the effectiveness of our results.
\end{abstract}

Keywords: nonstandard finite differences, unconditional positivity, stability, Lyapunov function.

\section{Introduction}

The application of theories of Differential Equations (DEs) in mathematical epidemiology has spread rapidly. Various epidemic models, which describe the dynamical behavior of the transmission of infectious disease, have been proposed in the literature (Enszer and Stadtherr, 2009; Ma et al., 2004; Kouche and Ainseba, 2010). The solutions of DEs describe the evolution of different classes of subpopulations in the model for different times. Therefore, one important task of mathematical modeling is to obtain accurate theoretical solutions (Jódar et al., 2008). However, theoretical solutions of these systems exist only occasionally and are usually complicated, so good approximations are necessary (Parra et al., 2010). Numerical methods are often the method of choice (Sekiguchi, 2009; Jang, 2007; Chinviriyasit and Chinviriyasit, 2010; Enatsu et al., 2010; Muroya et al., 2011; Jang and Elaydi, 2003). Furthermore, this allows better use of statistical data for numerical simulations since the infection data are compiled at discrete given time intervals (Enatsu et al., 2010).

The discrete models constructed by numerical methods yield a major difficulty in the calculation of numerical solutions, since they may cause Numerical Instabilities (NIs). NIs are solutions to the discrete equations that do not correspond to any solution of the original differential equations (Hildebrand, 1968;
Mickens, 1994). Discrete epidemic models contain parameters in addition to those already existing in the DEs such as the time and space step sizes. Variations in these extra parameters may cause bifurcation phenomena which show up as NIs. For example, traditional schemes like forward Euler, Runge-Kutta, and others sometimes generate oscillations and produce fictitious bifurcations, artificial chaos and false steady states (Lambert, 1973). Therefore, how to choose discrete schemes which guarantee the global dynamics of the models is a very important topic.

NSFD methods, developed by Mickens (1994; 2000; 2002; 2005), are increasingly being applied to numerical integration of differential equations (Alexander et al., 2006; Dumont and Lubuma, 2005; Bruggeman et al., 2007; Arenas et al., 2008; Moghadas et al., 2003; Anguelo and Lubuma, 2003; Dimitrov and Kojouharov, 2007; 2008; Dimitrov, 2005; Gumel, 2002; Jansen and Twizell, 2002; Chen and Clemence, 2006; Obaid et al., 2013). Their use is mainly based on the fact that they are very effective in preserving certain qualitative properties of the original differential equations (Mickens, 2000; Alexander et al., 2006; Sekiguchi and Ishiwata, 2010; Sekiguchi, 2010; Moghadas et al., 2003). Arenas et al. (2008) developed a non standard numerical scheme for an SIRS seasonal epidemiological model for RSV transmission. Sekiguchi and Ishiwata (2010) derived a discretized SIRS epidemic model with time delay by 
applying an NSFD scheme. With the NSFD scheme, Sekiguchi (2010) proved the permanence of an SIRS discrete epidemic model with time delays if an endemic equilibrium of each model exists. Moghadas et al. (2003) set forth a positivity preserving NSFD scheme for an epidemic model.

In this paper, we will show that a discrete epidemic model with vaccination constructed by NSFD unconditionally guarantee the positivity of solutions and the global dynamics of the corresponding continuous model.

The remainder of this paper is organized as follows. In the next section, we will introduce some useful definitions and a lemma for the continuous epidemic model with vaccination. In Section 3, we derive a discretized epidemic model with vaccination. We obtain a sufficient condition for the global asymptotic stability of the equilibria in Section 4. Numerical simulations are reported in Section 5.

\section{Preliminaries}

To prevent and control the spread of some communicable diseases, vaccination is one of the commonly used measures. Studies of epidemic models with vaccination have become an important issue in the mathematical theory of epidemiology (Ma et al., 2004; Anderson and May, 1991). Vaccine-induced immunity may wane in preventive vaccines against infectious diseases, such as hepatitis B, polio and mumps, and so forth. These vaccines can only offer a period of immunity to the diseases. For example, the immunization period of the hepatitis B vaccine is about five years, and that of the rabies vaccine is about one and half years. During the immunization period, the vaccinated individuals are subjected to the natural death rate.

Let $S(t)$ be the number of susceptible individuals, $I(t)$ be the number of infective individuals, and $R(t)$ be the number of removed individuals at time $t$. Ma et al. (2004) studied the following continuous epidemic model with vaccination, which is the following system of delay differential equations:

$$
\begin{aligned}
S^{\prime} & =a-d S-p S-\beta S I+p S(t-\tau) e^{-d \tau}, \\
I^{\prime} & =\beta S I-(d+\alpha+\gamma) I, \\
R^{\prime} & =\gamma I+p S-d R-p S(t-\tau) e^{-d \tau},
\end{aligned}
$$

where $a$ is the recruitment rate of the population, $d$ is the natural death rate of the population, $p$ is the vaccinating rate coefficient for a susceptible individual, $\beta$ is the infection coefficient, $\alpha$ is the disease-induced death rate coefficient, $\gamma$ is the recovery rate coefficient of the infected individuals. The immunization period is referred to as a delay $\tau$, which means those who are vaccinated at time $t-\tau$ would return to a susceptible individual at time $t$.

We adopt the following notation: $\mathbb{R}^{3}$ is a three-dimensional real Euclidean space with norm $|\cdot|$. For $\tau>0$, we denote by $C=C\left([-\tau, 0], \mathbb{R}^{3}\right)$ the Banach space of continuous functions mapping the interval $[-\tau, 0]$ into $\mathbb{R}^{3}$ with the topology of uniform convergence, i.e., for $\varphi=\left(\varphi_{1}, \varphi_{2}, \varphi_{3}\right) \in C$, the norm of $\varphi$ is defined as

$$
\|\varphi\|=\sup _{-\tau \leq \theta \leq 0}\left\{\left|\varphi_{1}(\theta)\right|,\left|\varphi_{2}(\theta)\right|,\left|\varphi_{3}(\theta)\right|\right\} .
$$

The initial conditions for the system (1) are given as

$$
\begin{aligned}
S(\theta) & =\varphi_{1}(\theta) \geq 0, \\
I(\theta) & =\varphi_{2}(\theta) \geq 0, \\
R(\theta) & =\varphi_{3}(\theta) \geq 0, \quad-\tau \leq \theta<0, \\
S(0) & =\varphi_{1}(0)>0, \\
I(0) & =\varphi_{2}(0)>0, \\
R(0) & =\varphi_{3}(0)>0
\end{aligned}
$$

and the solution of the system (1) is denoted by $(S(t), I(t), R(t))$.

Observe that the variable $R(t)$ does not appear in the first two equations of the system (1), and the behaviors of $R(t)$ can then be determined from the last equation in the system (1). This allows us to consider the reduced system

$$
\begin{aligned}
& S^{\prime}=a-d S-p S-\beta S I+p S(t-\tau) e^{-d \tau}, \\
& I^{\prime}=\beta S I-(d+\alpha+\gamma) I,
\end{aligned}
$$

where the initial conditions are

$$
\begin{aligned}
& S(\theta)=\varphi_{1}(\theta) \geq 0, \\
& I(\theta)=\varphi_{2}(\theta) \geq 0, \quad-\tau \leq \theta<0, \\
& S(0)=\varphi_{1}(0)>0, \\
& I(0)=\varphi_{2}(0)>0 .
\end{aligned}
$$

Set

$$
D=\left\{(S, I) \in \mathbb{R}^{2} \mid S>0, I>0, S+I \leq \frac{a}{d}\right\} .
$$

Let $\stackrel{\circ}{D}$ denote the interior of $D$. It can be verified that $D$ is positively invariant with respect to the system (3). Ma et al. (2004) derived the following result.

Lemma 1. In the system (3), $(S, I) \in D$. Set

$$
\begin{gathered}
S^{0}=\frac{a}{d+p\left(1-e^{-d \tau}\right)}, \\
S^{*}=\frac{d+\alpha+\gamma}{\beta}, \\
I^{*}=\frac{a}{d+\alpha+\gamma}\left(1-\frac{1}{R_{0}}\right)
\end{gathered}
$$

and

$$
R_{0}=\frac{\beta S^{0}}{d+\alpha+\gamma}
$$


Then, we have the following.

(i) If $R_{0} \leq 1$, then the system (3) has a unique diseasefree equilibrium $E^{0}=\left(S^{0}, 0\right)$ which is globally asymptotically stable in $D$.

(ii) If $R_{0}>1$, then the system (3) has two equilibria, a disease-free equilibrium $E^{0}$ and an endemic equilibrium $E^{*}=\left(S^{*}, I^{*}\right)$. The endemic equilibrium $E^{*}$ is globally asymptotically stable in $\stackrel{\circ}{D}$.

As is well known, it is desirable that the numerical schemes preserve the dynamics of the system (1). In particular, the numerical schemes must have at least the following properties (Mickens, 2005; Oran and Boris, 1987; Potter, 1973):

- Positivity of solutions: For any nonnegative initial data, the iterations must remain nonnegative.

- Correct number and stability of equilibria: The properties (i)-(ii) of the system (3) must hold. equation

Consider the nonlinear functional differential

$$
\frac{\mathrm{d} x(t)}{\mathrm{d} t}=f\left(x_{t}\right), \quad t \geq 0,
$$

where $f: C \rightarrow \mathbb{R}^{n}$ is continuous on compact subsets of $C$, and $x_{t} \in C$ is defined by $x_{t}(s)=x(t+s), s \in[-\tau, 0]$. Under this hypothesis, the solutions $x(t)=x(t, \phi)$ of Eqn. (5) are determined by the initial condition

$$
x_{t_{0}}=\phi, \quad \text { where } \phi \in C, t_{0}=0 .
$$

Elements of $x(t)$ and $f\left(x_{t}\right)$ will be denoted by $x^{i}(t)$ and $f^{i}\left(x_{t}\right), i=1,2, \ldots, n$ respectively. For any vector $x$, $x>0$ will be used to denote $x^{i}>0, \forall i$.

For numerical schemes, the time at integration step $n \in \mathbb{N}$ will be denoted by $t_{n}$. As this paper deals only with schemes using a fixed time step, the time step will be denoted by $h$, and $m$ will be a positive integer satisfying $\tau=m h$. The numerical approximation of the solution vector $x\left(t_{n}\right)$ will be denoted by $x_{n}$. According to Bruggeman et al. (2007) as well as Moghadas and Gumel (2003), we give the following definition.

Definition 1. (Unconditional positivity of a system) A system (5) is called unconditionally positive if $f\left(x_{t}\right)$ is such that $x(t)>0$ for all $t>0$, with $\phi \in C$ satisfying

$$
\phi(s) \geq 0, \quad s \in[-\tau, 0], \quad \phi(0)>0 .
$$

Definition 2. (Unconditional positivity of a numerical scheme) A numerical scheme $\psi$ for the system (5) is called unconditionally positive if $x_{n}>0, n=1,2, \ldots$ for any given $x_{k}=\phi(-k h)>0, k=0,1,2, \ldots, m$, with $\phi \in C$ satisfying (7).
Definition 3. (Unconditional stability of a numerical scheme) A numerical scheme $\Psi$ is called unconditionally stable if it is stable irrespective of the value of the step size used to simulate it.

Applying Mickens' nonstandard discretization to the continuous model (1), Ding et al. (2013), proposed the following discretized epidemic model with vaccination

$$
\begin{aligned}
\frac{S_{n+1}-S_{n}}{h}= & a-d S_{n}-p S_{n+1}-\beta S_{n+1} I_{n} \\
& +p S_{n-m} e^{-d \tau}, \\
\frac{I_{n+1}-I_{n}}{h}= & \beta S_{n+1} I_{n}-(d+\alpha+\gamma) I_{n}, \\
\frac{R_{n+1}-R_{n}}{h}= & \gamma I_{n}+p S_{n+1}-d R_{n}-p S_{n-m} e^{-d \tau} .
\end{aligned}
$$

There, they show that, when

$$
h<h_{0}=\frac{1}{d+\alpha+\gamma},
$$

the difference equation system (8) can keep the global dynamics of the continuous system (1). However, the results in Ding et al. (2013) are limited by the step size. In this paper, this restriction is removed by constructing more proper nonstandard scheme.

\section{Unconditionally positive discretized model}

In this section, denote by $S_{n}$ the susceptible class, by $I_{n}$ the infective class and by $R_{n}$ the recovered class at the $n$-th step, respectively. Applying Mickens' nonstandard discretization to the continuous model (1), we derive the following discretized epidemic model with vaccination, which is a system of difference-delay equations:

$$
\begin{aligned}
\frac{S_{n+1}-S_{n}}{h}= & a-d S_{n+1}-p S_{n+1}-\beta S_{n+1} I_{n} \\
& +p S_{n-m} e^{-d \tau}, \\
\frac{I_{n+1}-I_{n}}{h}= & \beta S_{n+1} I_{n}-(d+\alpha+\gamma) I_{n+1}, \\
\frac{R_{n+1}-R_{n}}{h}= & \gamma I_{n+1}+p S_{n+1}-d R_{n+1} \\
& -p S_{n-m} e^{-d \tau},
\end{aligned}
$$

where $n=0,1, \ldots$ The initial conditions of the system (9) are given by

$$
\begin{aligned}
& S(-k)=\varphi_{1}(-k h) \geq 0, \\
& S(0)>0, \quad I(0)>0, \\
& R(0)>0, \quad k=1,2, \ldots, m .
\end{aligned}
$$

Observe that the variable $R_{n}$ does not appear in the first two equations of the system (9), and the behaviors of 
$R_{n}$ can then be determined from the last equation in the system (9). This allows us to consider the reduced system

$$
\begin{aligned}
\frac{S_{n+1}-S_{n}}{h} & =a-(d+p) S_{n+1}-\beta S_{n+1} I_{n} \\
& +p S_{n-m} e^{-d \tau}, \\
\frac{I_{n+1}-I_{n}}{h} & =\beta S_{n+1} I_{n}-(d+\alpha+\gamma) I_{n+1},
\end{aligned}
$$

where the initial conditions are

$$
\begin{array}{r}
S(-k) \geq 0, \quad S(0)>0, \quad I(0)>0, \\
k=1,2, \ldots, m .
\end{array}
$$

After some simple manipulations, the system (11) is transformed into the following form:

$$
\begin{aligned}
S_{n+1} & =\frac{h a+S_{n}+h p S_{n-m} e^{-d \tau}}{1+(d+p) h+\beta h I_{n}}, \\
I_{n+1} & =\frac{\beta h S_{n+1} I_{n}+I_{n}}{[1+h(d+\alpha+\gamma)]} .
\end{aligned}
$$

It is easy to get the following lemma.

Lemma 2. The system (11) with the initial condition (12) is unconditionally positive.

$$
\begin{aligned}
& \text { Set } N_{n}=S_{n}+I_{n}+R_{n} \text {. Then } \\
& N_{n+1}=\frac{a h+N_{n}-\alpha h I_{n+1}}{1+d h} \leq \frac{a h+N_{n}}{1+d h} .
\end{aligned}
$$

Therefore,

$$
N_{n} \leq \max \left\{N_{0}, \frac{a}{d}\right\}
$$

and

$$
\limsup _{n \rightarrow \infty} N_{n} \leq \frac{a}{d}
$$

Theorem 1. For any solution $\left(S_{n}, I_{n}, R_{n}\right)$ of the system (9), the total population $N_{n}$ satisfies

$$
\limsup _{n \rightarrow \infty} N_{n} \leq \frac{a}{d}
$$

Define

$$
\begin{aligned}
\Gamma=\left\{\left(S_{n}, I_{n}\right) \in \mathbb{R}^{2} \mid 0\right. & <S_{n}, \\
& \left.0<I_{n}, 0<S_{n}+I_{n} \leq \frac{a}{d}\right\},
\end{aligned}
$$

and let $\stackrel{\circ}{\Gamma}$ denote the interior of $\Gamma$. It can be verified that $\Gamma$ is positively invariant with respect to the system (11). Our results will be stated for the system (11) in $\Gamma$.

\section{Global asymptotic stability of the equilibria}

In this section, we will show that the system (11) is unconditionally stable.

An equilibrium of the system (11) satisfies

$$
\begin{aligned}
& 0=a-(d+p) S-\beta S I+p S e^{-d \tau}, \\
& 0=\beta S I-(d+\alpha+\gamma) I .
\end{aligned}
$$

It is easy to verify that the equilibria of the discretized system (9) are the equilibria of the system (1). Furthermore, the equilibria of the system (11) are the same as (3), namely, $E^{0}$ and $E^{*}$.

In this section, firstly, we obtain a sufficient condition for global asymptotic stability of the disease-free equilibrium $E^{0}$ in the system (11) by a Lyapunov function.

Theorem 2. In the system (11), we have the following:

(i) If $R_{0} \leq 1$, the disease-free equilibrium $E^{0}=\left(S^{0}, 0\right)$ of the system (11) is globally asymptotically stable in $\Gamma$.

(ii) If $R_{0}>1, E^{0}$ is unstable.

Proof. Part (i). It is convenient to translate the disease-free equilibrium $E^{0}$ to the origin by setting $X_{n}=$ $S_{n}-S^{0}$. By Eqn. 16), we know $S^{0}$ satisfies $a-(d+$ p) $S^{0}+p S^{0} e^{-d \tau}=0$. Then Eqn. (111) becomes

$$
\begin{aligned}
\frac{X_{n+1}-X_{n}}{h}= & -d X_{n+1}-p X_{n+1}-\beta X_{n+1} I_{n} \\
& -\beta S^{0} I_{n}+p X_{n-m} e^{-d \tau}, \\
\frac{I_{n+1}-I_{n}}{h}= & \beta X_{n+1} I_{n}+\beta S^{0} I_{n} \\
& -(d+\alpha+\gamma) I_{n+1} .
\end{aligned}
$$

Define

$$
\left.L\right|_{(11) n} ^{1}=\frac{X_{n}^{2}}{2}+[1+(d+\alpha+\gamma) h] S^{0} I_{n} .
$$

We obtain

$$
\begin{aligned}
&\left.\Delta L\right|_{(11) n} ^{1} \\
&= \frac{1}{2}\left(X_{n+1}^{2}-X_{n}^{2}\right)+\left[h \beta S^{0} X_{n+1} I_{n}\right. \\
&\left.+h \beta S^{0^{2}} I_{n}-(d+\alpha+\gamma) h S^{0} I_{n}\right] \\
&= \frac{1}{2}\left\{X_{n+1}^{2}-\left[X_{n+1}+\left((d+p) h X_{n+1}\right.\right.\right. \\
&\left.\left.\left.+\beta h X_{n+1} I_{n}+\beta h S^{0} I_{n}-p h X_{n-m} e^{-d \tau}\right)\right]^{2}\right\}
\end{aligned}
$$




$$
\begin{aligned}
& +S^{0}\left(\beta h X_{n+1} I_{n}+\beta h S^{0} I_{n}-(d+\alpha+\gamma) h I_{n}\right) \\
\leq & -X_{n+1}\left((d+p) h X_{n+1}+\beta h X_{n+1} I_{n}\right. \\
& \left.+\beta h S^{0} I_{n}-p h X_{n-m} e^{-d \tau}\right)+S^{0}\left(\beta h X_{n+1} I_{n}\right. \\
& \left.+\beta h S^{0} I_{n}-(d+\alpha+\gamma) h I_{n}\right) \\
= & -(d+p) h X_{n+1}^{2}-\beta h X_{n+1}^{2} I_{n} \\
& +p h X_{n+1} X_{n-m} e^{-d \tau}+\beta h S^{0^{2}} I_{n} \\
& -(d+\gamma+\alpha) h S^{0} I_{n} .
\end{aligned}
$$

Then

$$
\begin{aligned}
\left.\Delta L\right|_{(11) n} ^{1} \leq & -(d+p) h X_{n+1}^{2}-\beta h X_{n+1}^{2} I_{n} \\
& +\frac{p h e^{-d \tau}}{2} X_{n+1}^{2}+\frac{p h e^{-d \tau}}{2} X_{n-m}^{2} \\
& +\beta h S^{0^{2}} I_{n}-(d+\alpha+\gamma) h S^{0} I_{n}
\end{aligned}
$$

Define

$$
\left.L\right|_{(11) n} ^{2}=\frac{h p e^{-d \tau}}{2} \sum_{k=n-m}^{n} X_{k}^{2} .
$$

Similarly, we obtain

$$
\begin{aligned}
\left.\Delta L\right|_{(11) n} ^{2} & =\frac{h p e^{-d \tau}}{2}\left(\sum_{k=n+1-m}^{n+1} X_{k}^{2}-\sum_{k=n-m}^{n} X_{k}^{2}\right) \\
& =\frac{h p e^{-d \tau}}{2}\left(X_{n+1}^{2}-X_{n-m}^{2}\right) .
\end{aligned}
$$

Set $\left.L\right|_{(11) n}=\left.L\right|_{(11) n} ^{1}+\left.L\right|_{(11) n} ^{2}$. Then we get

$$
\begin{aligned}
&\left.\Delta L\right|_{(11) n} \\
&=\left.\Delta L\right|_{(11) n} ^{1}+\left.\Delta L\right|_{(11) n} ^{2} \\
& \leq-\left[d+p\left(1-e^{-d \tau}\right)\right] h X_{n+1}^{2}-\beta h X_{n+1}^{2} I_{n} \\
&+\beta h S^{0^{2}} I_{n}-(d+\alpha+\gamma) h S^{0} I_{n} \\
& \leq-\left[d+p\left(1-e^{-d \tau}\right)\right] h X_{n+1}^{2} \\
&-S^{0} h\left(d+\alpha+\gamma-\beta S^{0}\right) I_{n} \\
& \leq-\left[d+p\left(1-e^{-d \tau}\right)\right] h X_{n+1}^{2} \\
&-S^{0} h(d+\alpha+\gamma)\left(1-R_{0}\right) I_{n} .
\end{aligned}
$$

Obviously, when $R_{0} \leq 1,\left.\Delta L\right|_{(11) n} \leq 0$; when $R_{0}<1$, $\left.\Delta L\right|_{(11) n}=0 \Leftrightarrow X_{n+1}=X_{n-m}=0, I_{n}=0$; when $R_{0}=1,\left.\Delta L\right|_{(11) n}=0 \Leftrightarrow X_{n+1}=X_{n-m}=$ 0 . Substituting $R_{0}=1$ and $X_{n+1}=X_{n-m}=0$ into the system (17), we have $I_{n}=I_{n+1}$. That is, the only compact invariant set where $\left\{\left.\triangle L\right|_{(11) n}=0\right\}$ is the singleton $\left\{X_{n}=0, I_{n}=0\right\}$. By Lyapunov functions and stability (Stuart and Humphries, 1996), we have that $E^{0}$ is globally asymptotically stable in $\Gamma$, if $R_{0} \leq 1$.

Part (ii). If $R_{0}>1$, the linearization of Eqn. (17) at $E^{0}$ is

$$
\begin{aligned}
\frac{X_{n+1}-X_{n}}{h}= & -d X_{n+1}-p X_{n+1}-\beta S^{0} I_{n} \\
& +p X_{n-m} e^{-d \tau}, \\
\frac{I_{n+1}-I_{n}}{h}= & \beta S^{0} I_{n}-(d+\alpha+\gamma) I_{n+1} .
\end{aligned}
$$

Then the characteristic equation takes the form

$$
\left|\begin{array}{cc}
\tilde{\vartheta}_{1} & \beta h S^{0} \lambda^{m} \\
0 & \tilde{\vartheta}_{2}
\end{array}\right|=0
$$

where

$$
\begin{gathered}
\tilde{\vartheta}_{1}=[1+(d+p) h] \lambda^{m+1}-\lambda^{m}-p h e^{-d \tau}, \\
\tilde{\vartheta}_{2}=\lambda^{m+1}-\frac{1+(\alpha+d+\gamma) R_{0} h}{1+(\alpha+d+\gamma) h} \lambda^{m} .
\end{gathered}
$$

It is easy to demonstrate that

$$
\lambda_{0}=\frac{1+(\alpha+d+\gamma) R_{0} h}{1+(\alpha+d+\gamma) h}
$$

is one of the roots of Eqn. (25). Clearly, if $R_{0}>1, \lambda_{0}>$ 1. Therefore, if $R_{0}>1$, the disease-free equilibrium $E^{0}$ of the system (11) is unstable.

Next, we will show the global stability for the endemic equilibrium $E^{*}$ of the system (11) by a Lyapunov function.

Theorem 3. If $R_{0}>1$, the endemic equilibrium $E^{*}=$ $\left(S^{*}, I^{*}\right)$ of the system (11) is globally asymptotically stable in $\stackrel{\circ}{\Gamma}$.

Proof. Set

$$
\left.V\right|_{(11) n}=\left.V\right|_{(11) n} ^{1}+\left.V\right|_{(11) n} ^{2}
$$

where

$$
\begin{aligned}
\left.V\right|_{(11) n} ^{1}= & S_{n}-S^{*}-S^{*} \ln \frac{S_{n}}{S^{*}} \\
+ & {[1+(\alpha+d+\gamma) h] } \\
& \times\left(I_{n}-I^{*}-I^{*} \ln \frac{I_{n}}{I^{*}}\right), \\
\left.V\right|_{(11) n} ^{2}= & p h e^{-d \tau} \sum_{k=-m}^{0}\left(S_{n-k}-S^{*}\right. \\
& \left.-S^{*} \ln \frac{S_{n-k}}{S^{*}}\right) .
\end{aligned}
$$

Set $\psi(x)=1+\ln x-x$ for all $x>0$. We know that

$$
\psi(x) \leq 0 \text { for } x>0,
$$


with the equality holding if and only if $x=1$. Then we get

$$
\begin{aligned}
\Delta V & \left.\right|_{(11) n} ^{1} \\
= & {\left[S_{n+1}-S_{n}+S^{*} \ln \frac{S_{n}}{S_{n+1}}\right] } \\
& +[1+(\alpha+d+\gamma) h]\left[I_{n+1}-I_{n}+I^{*} \ln \frac{I_{n}}{I_{n+1}}\right] \\
= & {\left[S_{n+1}-S_{n}+S^{*}\left(\psi\left(\frac{S_{n}}{S_{n+1}}\right)-1+\frac{S_{n}}{S_{n+1}}\right)\right] } \\
& +[1+(\alpha+d+\gamma) h]\left[I_{n+1}-I_{n}\right. \\
& \left.+I^{*}\left(\psi\left(\frac{I_{n}}{I_{n+1}}\right)-1+\frac{I_{n}}{I_{n+1}}\right)\right] \\
\leq & \left(1-\frac{S^{*}}{S_{n+1}}\right)\left(S_{n+1}-S_{n}\right) \\
& +[1+(\alpha+d+\gamma) h]\left[\left(1-\frac{I^{*}}{I_{n+1}}\right)\left(I_{n+1}-I_{n}\right)\right] .
\end{aligned}
$$

Together with Eqns. (11) and (16), we produce

$$
\begin{aligned}
\Delta V & \left.\right|_{(11) n} ^{1} \\
\leq & \left(1-\frac{S^{*}}{S_{n+1}}\right)\left[(d+p) h S^{*}+\beta h S^{*} I^{*}-p h S^{*} e^{-d \tau}\right. \\
& \left.-(d+p) h S_{n+1}-\beta h S_{n+1} I_{n}+p h S_{n-m} e^{-d \tau}\right] \\
& +\left(1-\frac{I^{*}}{I_{n+1}}\right)\left[\beta h S_{n+1} I_{n}-(d+\gamma+\alpha) h I_{n}\right] .
\end{aligned}
$$

Similarly, we have

$$
\begin{array}{rl}
\left.\Delta V\right|_{(11) n} ^{2} & p h e^{-d \tau} \sum_{k=-m}^{0}\left(S_{n+1-k}-S_{n-k}+S^{*} \ln \frac{S_{n-k}}{S_{n+1-k}}\right) \\
= & p h e^{-d \tau}\left(S_{n+1}-S_{n-m}+S^{*} \ln \frac{S_{n-m}}{S_{n+1}}\right) \\
= & p h e^{-d \tau}\left[S_{n+1}-S_{n-m}\right. \\
& \left.+S^{*}\left(\psi\left(\frac{S_{n-m}}{S_{n+1}}\right)-1+\frac{S_{n-m}}{S_{n+1}}\right)\right] \\
\leq & p h e^{-d \tau}\left(S_{n+1}-S_{n-m}-S^{*} \frac{S_{n+1}-S_{n-m}}{S_{n+1}}\right) .
\end{array}
$$

By Eqns. 16, 29) and (30), and using the fact that

$$
\frac{S^{*}}{S_{n+1}}+\frac{S_{n+1}}{S^{*}} \geq 2,
$$

with the equality holding if and only if $S^{*}=S_{n+1}$, we obtain

$$
\begin{aligned}
&\left.\Delta V\right|_{(11) n} \\
&=\left.\Delta V\right|_{(11) n} ^{1}+\left.\Delta V\right|_{(11) n} ^{2} \\
& \leq h S^{*}\left[d+p\left(1-e^{-d \tau}\right)\right]\left(2-\frac{S^{*}}{S_{n+1}}-\frac{S_{n+1}}{S^{*}}\right) \\
&+\left(\beta h S^{*} I^{*}-\beta h S_{n+1} I_{n}-\beta h \frac{S^{*^{2}} I^{*}}{S_{n+1}}+\beta h S^{*} I_{n}\right) \\
&+\left(1-\frac{I^{*}}{I_{n+1}}\right)\left[\beta h S_{n+1} I_{n}-(d+\alpha+\gamma) h I_{n}\right] \\
& \leq \beta h\left[S^{*} I^{*}-S_{n+1} I_{n}-\frac{S^{*^{2}} I^{*}}{S_{n+1}}+S^{*} I_{n}\right. \\
&\left.+\left(1-\frac{I^{*}}{I_{n+1}}\right)\left(S_{n+1} I_{n}-S^{*} I_{n}\right)\right] \\
&= \beta h\left(S^{*} I^{*}-\frac{S^{*} I^{*}}{S_{n+1}}-\frac{S_{n+1} I_{n}}{I_{n+1}} I^{*}+\frac{S^{*} I^{*} I_{n}}{I_{n+1}}\right) \\
&= \beta h I^{*}\left(\frac{S^{*}}{S_{n+1}}-\frac{I_{n}}{I_{n+1}}\right)\left(S_{n+1}-S^{*}\right) \\
&= I^{*}\left(1+\beta h S^{*}\right)\left(\frac{S^{*}}{S_{n+1}}-\frac{I_{n}}{I_{n+1}}\right)\left(\frac{I_{n+1}}{I_{n}}-1\right) .
\end{aligned}
$$

From Eqn. (11), it is easy to see that

$$
I_{n+1}-I_{n}=\frac{1}{1+\beta S^{*} h}\left(\beta h S_{n+1} I_{n}-\beta S^{*} h I_{n}\right) .
$$

By Eqn. (32), we get

$$
\begin{aligned}
& \left(1+\beta S^{*} h\right)\left(\frac{I_{n+1}}{I_{n}}-1\right)>-\beta h S^{*}, \\
& \frac{S^{*}}{S_{n+1}}=\frac{\beta h S^{*}}{\left(\frac{I_{n+1}}{I_{n}}-1\right)\left(1+\beta S^{*} h\right)+\beta h S^{*}}
\end{aligned}
$$

Set $x=I_{n+1} / I_{n}$. Then, by Eqn. (33), we have

$$
\begin{aligned}
& \left.\Delta V\right|_{(11) n} \\
& \leq I^{*}\left(1+\beta h S^{*}\right) \\
& \quad \times\left[\frac{\beta h S^{*}}{(x-1)\left(1+\beta S^{*} h\right)+\beta h S^{*}}-\frac{1}{x}\right](x-1) \\
& =-\frac{I^{*}\left(1+\beta h S^{*}\right)(x-1)^{2}}{x\left[(x-1)\left(1+\beta S^{*} h\right)+\beta h S^{*}\right]} \leq 0
\end{aligned}
$$

According to Eqns. (34), 28) and (31), we know $\left.\Delta V\right|_{(11) n}=0 \Leftrightarrow S_{n+1}=S_{n}=S_{n-m}=S^{*}$ and $I_{n+1}=I_{n}$. Substituting this into Eqn. 111, we get the only compact invariant set, where $\left\{\left.\Delta V\right|_{(11) n}=0\right\}$ is the singleton $\left\{E^{*}\right\}$. By LaSalle's invariance principle (Sundarapandian, 2003), $E^{*}$ is globally asymptotically stable in $\Gamma^{\circ}$.

Remark 1. From Theorems 2 and 3 , it cannot happen that $S_{n}$ is exponential decay. Thus, by the third equation of (9), it should be easy to show that $R_{n}>0$ with the initial 
condition (12). Together with Lemma 2, we know our results in this paper can be translated straightforwardly to the system (9).

\section{Numerical simulation}

For the system (9), Theorem 2 implies that the disease decays if $R_{0} \leq 1$, and Theorem 3 implies that the disease persists if $R_{0}>1$. In order to illustrate the validity of our results, we consider the following two epidemic models with vaccination. Firstly,

$$
\begin{aligned}
S^{\prime}= & 14-0.3 S-0.6 S-0.04 S I \\
& +0.6 S(t-10) e^{-0.3 \times 10} \\
I^{\prime}= & 0.04 S I-(0.3+0.009+0.9) I \\
R^{\prime}= & 0.9 I+0.6 S-0.3 R \\
& -0.6 S(t-10) e^{-0.3 \times 10}
\end{aligned}
$$

After some simple manipulations, we get $R_{0}=0.5276<$ 1 and Eqn. (35) has a unique equilibrium $E^{0}=$ $\left(S^{0}, I^{0}, R^{0}\right)=(15.95,0,30.72)$. By Lemma 1 i $), E^{0}$ is globally asymptotically stable.

Then

$$
\begin{aligned}
S^{\prime}= & 10-0.4 S-0.5 S-0.5 S I \\
& +0.5 S(t-10) e^{-0.4 \times 10}, \\
I^{\prime}= & 0.5 S I-(0.4+0.4+0.6) I, \\
R^{\prime}= & 0.6 I+0.5 S-0.4 R \\
& -0.5 S(t-10) e^{-0.4 \times 10} .
\end{aligned}
$$

It is easy to see that $R_{0}=3.9955>1$ and Eqn. (35) has two equilibria,

$$
\begin{aligned}
& E^{0}=\left(S^{0}, I^{0}, R^{0}\right)=(11.1874,0,13.8126), \\
& E^{*}=\left(S^{*}, I^{*}, R^{*}\right)=(2.8,5.3551,11.4897) .
\end{aligned}
$$

By Lemma1(ii), $E^{*}$ is globally asymptotically stable.

In Figs. 16 the numbers of susceptible, infective and recovered individuals (on the vertical axis) are plotted versus the time steps $n$ (on the horizontal axis). Figure 1 shows that the solutions of the system (35) under the numerical schemes (9) converge to the disease-free equilibrium when $h=0.1,1,11$. We can see that the disease free equilibrium $E^{0}$ of the system (35) is globally asymptotically stable. On the other hand, Fig. 4 shows that the solutions of the system (36) under the method (9) converge to the endemic equilibrium when $h=0.1,1,11$, and indicates that $E^{*}$ of the system 36 is globally asymptotically stable.

In the following, we give some comparison of the numerical scheme (8) with the scheme (9). Firstly, we use the NSFD method (8) to calculate numerical solutions for the underlying system (1). With the system (35), moreover, when the step size reaches a certain degree, the numerical solution exhibits oscillations of period two (see Fig. 2(a)), and even completely loses stability (see Fig. 3(a). With the growth of the step size, the situation of the system (36) is analogous to that of the system (35) (see Figs. 5(a) and 6(a). Then, we also use the NSFD method (9) with the systems (35) and (36). It is shown that the stability of the disease free equilibrium $E^{0}$ and endemic equilibrium $E^{*}$ have not been influenced even though the step size grows (see Figs, 1(c), 2(b), 3(b) and 4(c) 5(b) 6(b).

In addition, we make a comparison between the 4-th order Runge-Kutta method and the numerical scheme (9) for the system (35). It can be seen that the 4-th order Runge-Kutta method did not converge (see Fig 7(b) or in some cases took unreal negative values (see Fig.7(a) for the infective population, while the numerical scheme (9) converges to the correct endemic point and only has positive solutions with the same time step size.

In order to further illustrate the advantages of the proposed numerical scheme (9), we take the following parameter values: $a=0.002, p=1, d=0.002$, $b=500, \alpha=20$ and $\gamma=1$. In Fig. 8 for $R_{0}>1$, it can be observed that the numerical scheme (9) converges correctly to the endemic equilibrium point and only produces positive values for all times $t$. However, the routines of the Matlab software program with their default error tolerance did not converge.

\section{Conclusion and discussion}

In this paper, we derived a discretized epidemic model with vaccination by the NSFD method. For any $h>0$, we showed the positivity of numerical solutions and obtained sufficient conditions for global behaviors of the equilibria by a Lyapunov function. Our results demonstrate that the dynamic behaviors of a discretized system is in keeping with the continuous one.

\section{Acknowledgment}

This work was supported by the National Natural Science Foundation of China (11271101) and the NNSF of the Shandong Province (ZR2012AQ027), the Natural Scientific Research Innovation Foundation in the Harbin Institute of Technology (HIT NSRIF 2011095) and the Scientific Research Foundation of the Harbin Institute of Technology at Weihai (HIT (WH) XB201124).

The authors would like to thank the anonymous referees and the editor for their valuable remarks and comments which have improved the quality of our study.

\section{References}

Alexander, M.E., Summers, A.R. and Moghadas, S.M. (2006). Neimark-Sacker bifurcations in a non-standard numerical 


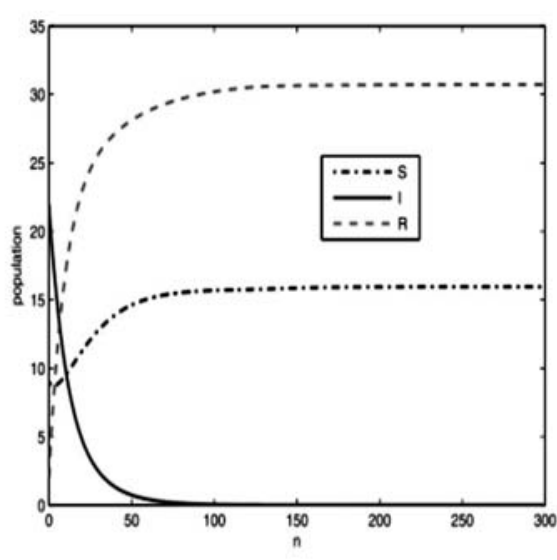

(a)

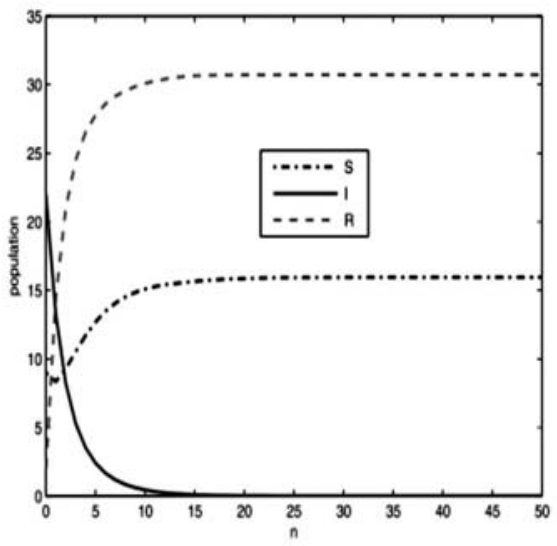

(b)

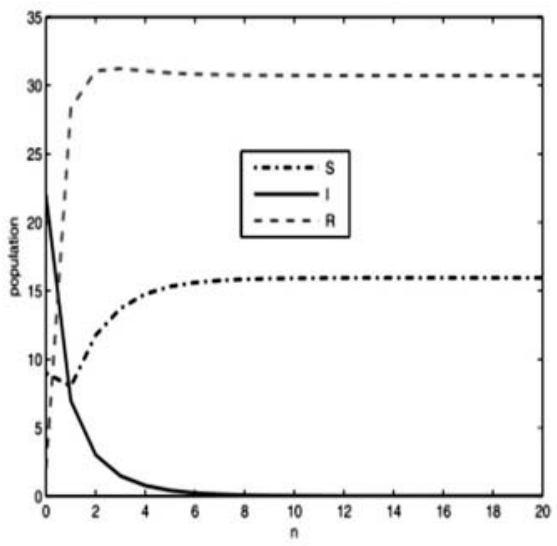

(c)

Fig. 1. Solution of the system (35) under the numerical scheme 9): $h=0.1$ (a), $h=1$ (b) and $h=11$ (c).

scheme for a class of positivity-preserving ODEs, Proceedings of the Royal Society, A: Mathematical, Physical and Engineering Sciences 462(2074): 3167-3184.

Anderson, R. and May, R. (1991). Infectious Diseases of Hu-

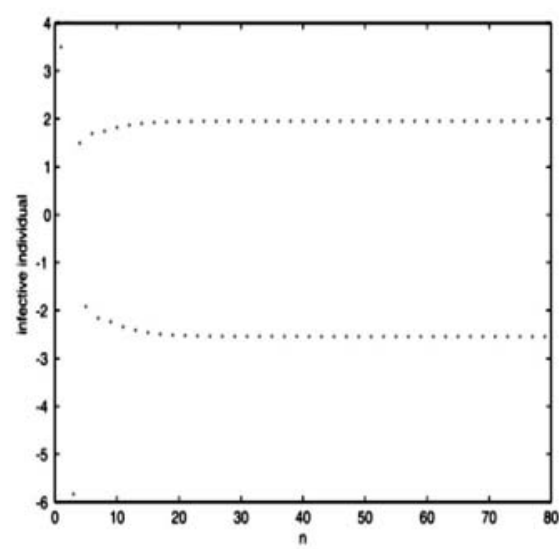

(a)

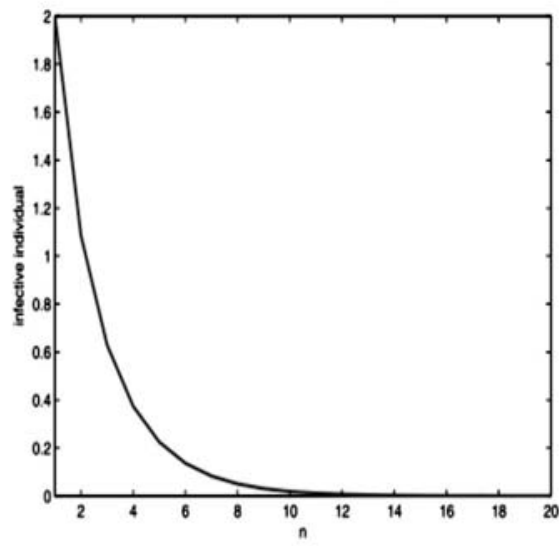

(b)

Fig. 2. Solution of the system $(35)$ with $h=3.67$ on the $(I, n)$ plane: under the numerical scheme (8) (a), under the numerical scheme 9) (b).

mans: Dynamics and Control, Oxford University Press, Oxford/New York, NY.

Anguelo, V.R. and Lubuma, J. (2003). Nonstandard finite difference method by nonlocal approximation, Mathematics and Computers in Simulation 61(3): 465-475.

Arenas, A., Moraño, J. and Cortés, J. (2008). Non-standard numerical method for a mathematical model of RSV epidemiological transmission, Computers and Mathematics with Applications 56(3): 670-678.

Bruggeman, J., Burchard, H., Kooi, B.W. and Sommeijer, B. (2007). A second-order, unconditionally positive, mass-conserving integration scheme for biochemical systems, Applied Numerical Mathematics 57(1): 36-58.

Chen, M. and Clemence, D. (2006). Stability properties of a nonstandard finite difference scheme for a hantavirus epidemic model, Journal of Difference Equations and Applications 12(12): 1243-1256.

Chinviriyasit, S. and Chinviriyasit, W. (2010). Numerical modelling of an SIR epidemic model with diffusion, Applied Mathematics and Computation 216(2): 395-409. 


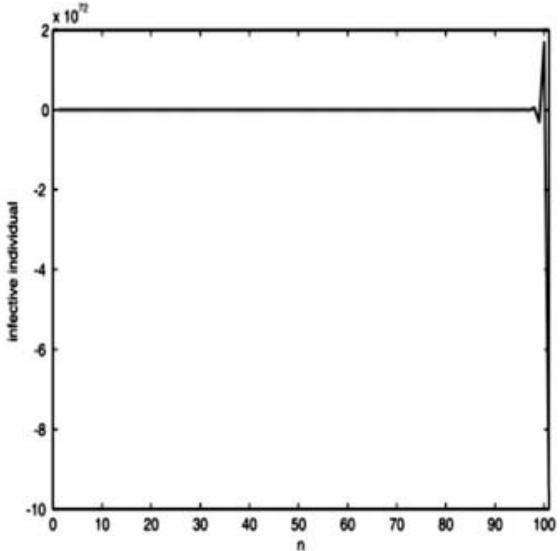

(a)

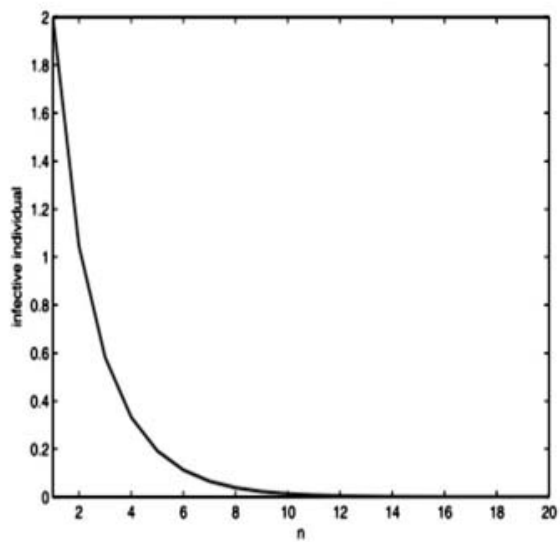

(b)

Fig. 3. Solution of the system (35) with $h=5.5$ on the $(I, n)$ plane: under the numerical scheme 8 (a), under the numerical scheme 9) (b).

Dimitrov, D.T. and Kojouharov, H. (2005). Nonstandard finite-difference schemes for general two-dimensional autonomous dynamical systems, Applied Mathematics Letters 18(7): 769-774.

Dimitrov, D. and Kojouharov, H. (2007). Stability-preserving finite-difference methods for general multi-dimensional autonomous dynamical systems, International Journal of Numerical Analysis and Modeling 4(2): 280-290.

Dimitrov, D. and Kojouharov, H. (2008). Nonstandard finite difference methods for predator-prey models with general functional response, Mathematics and Computers in Simulation 78(1): 1-11.

Ding, D., Ma, Q. and Ding, X. (2013). A non-standard finite difference scheme for an epidemic model with vaccination, Journal of Difference Equations and Applications 19(2): 179-190.

Dumont, Y. and Lubuma, J.M.-S. (2005). Non-standard finite-difference methods for vibro-impact problems, Proceedings of the Royal Society, A: Mathematical, Physical and Engineering Sciences 461(2058): 1927-1950.

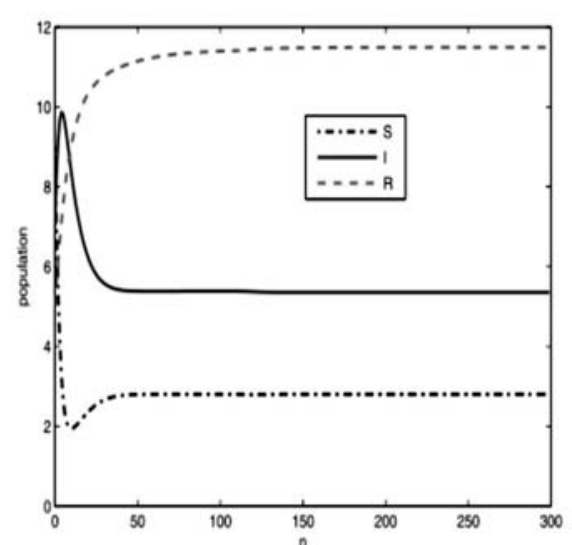

(a)

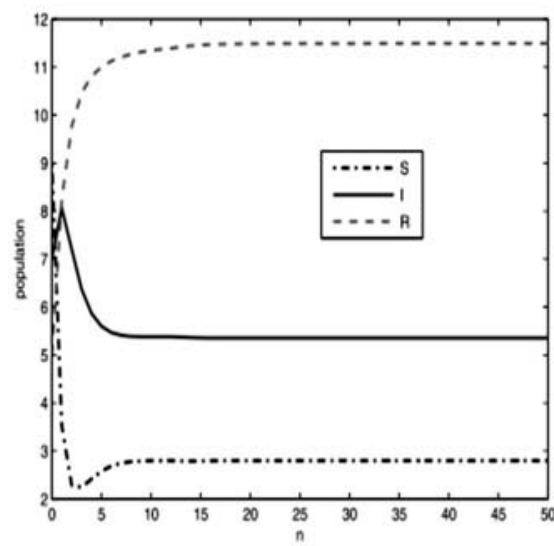

(b)

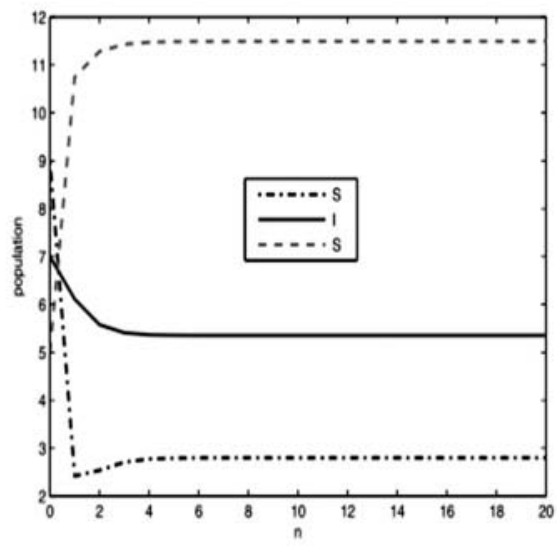

(c)

Fig. 4. Solution of the system (36) under the numerical scheme 9): $h=0.1$ (a), $h=1$ (b), $h=11$ (c).

Enatsu, Y., Nakata, Y. and Muroya, Y. (2010). Global stability for a class of discrete SIR epidemic models, Mathematical Biosciences and Engineering 7: 347-361.

Enszer, J.A. and Stadtherr, M.A. (2009). Verified solution 


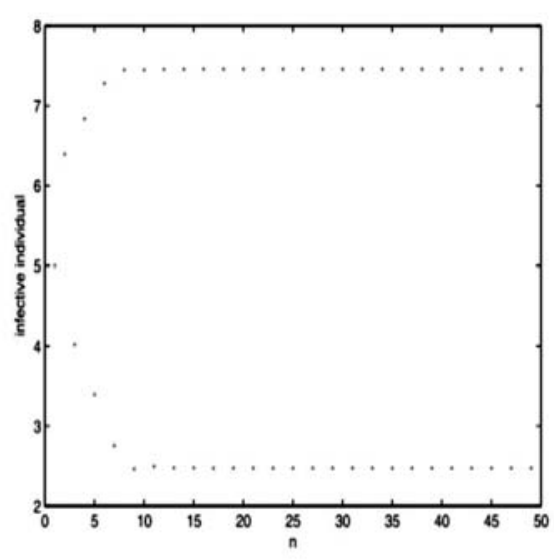

(a)

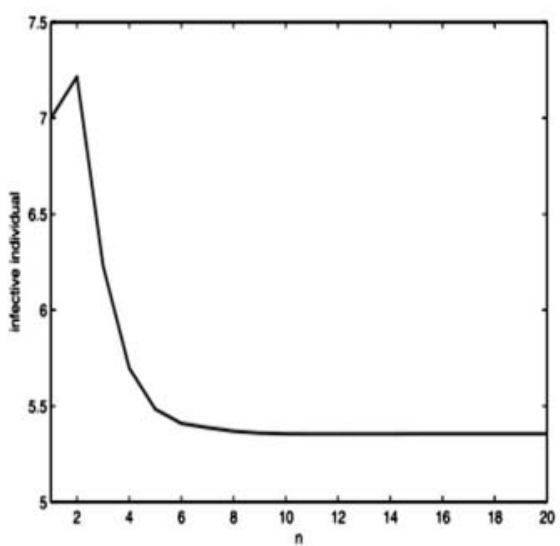

(b)

Fig. 5. Solution of the system (36) with $h=2.2$ on the $(I, n)$ plane: under the numerical scheme (8) (a), under the numerical scheme 9) (b).

method for population epidemiology models with uncertainty, International Journal of Applied Mathematics and Computer Science 19(3): 501-512, DOI: 10.2478/v10006-009-0040-4.

Gumel, A. (2002). A competitive numerical method for a chemotherapy model of two HIV subtypes, Applied Mathematics and Computation 131(2): 329-337.

Hildebrand, F. (1968). Finite Difference Equations and Simulations, Prentice-Hall, Englewood Cliffs, NJ.

Jódar, L., Villanueva, R., Arenas, A. and González, G. (2008). Nonstandard numerical methods for a mathematical model for influenza disease, Mathematics and Computers in Simulation 79(3): 622-633.

Jang, S. (2007). On a discrete west Nile epidemic model, Computational and Applied Mathematics 26(3): 397-414.

Jang, S. and Elaydi, S. (2003). Difference equations from discretization of a continuous epidemic model with immigration of infectives, Canadian Applied Mathematics Quarterly 11(1): 93-105.

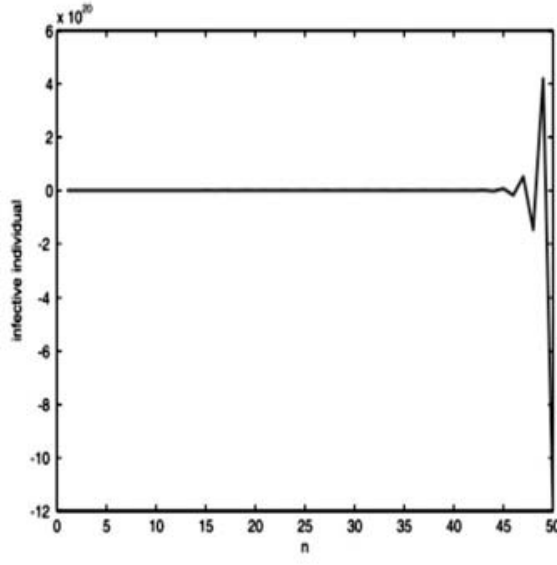

(a)

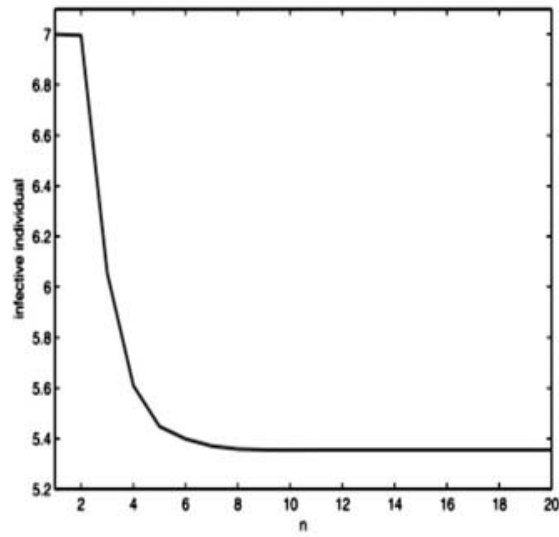

(b)

Fig. 6. Solution of the system 36 with $h=2.75$ on the $(I, n)$ plane: under the numerical scheme (8) (a), under the numerical scheme 9) (b).

Jansen, H. and Twizell, E. (2002). An unconditionally convergent discretization of the SEIR model, Mathematics and Computers in Simulation 58(2): 147-158.

Kouche, M. and Ainseba, B. (2010). A mathematical model of HIV-1 infection including the saturation effect of healthy cell proliferation, International Journal of Applied Mathematics and Computer Science 20(3): 601-612, DOI: 10.2478/v10006-010-0045-z.

Lambert, J. (1973). Computational Methods in Ordinary Differential Equations, Wiley, New York, NY.

Ma, Z., Zhou, Y., Wang, W. and Jing, Z. (2004). The Mathematical Modeling and Study of the Dynamics of Infectious Diseases, Science Press, Beijing.

Mickens, R. (1994). Nonstandard Finite Difference Models of Differential Equations, World Scientific, Singapore.

Mickens, R. (2000). Advances in the Applications of Nonstandard Finite Difference Schemes, World Scientific, Singapore. 


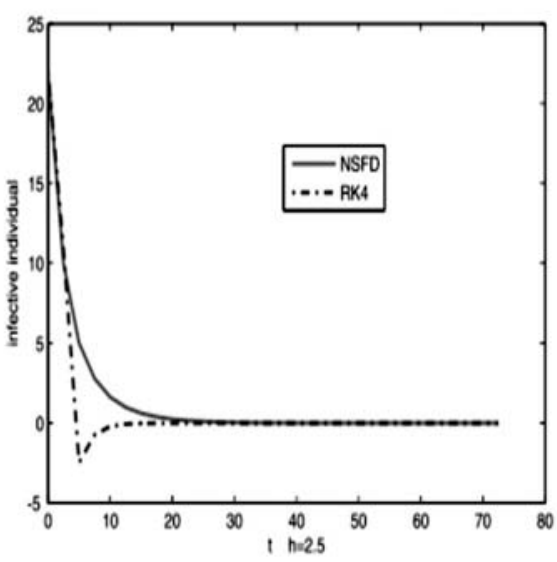

(a)

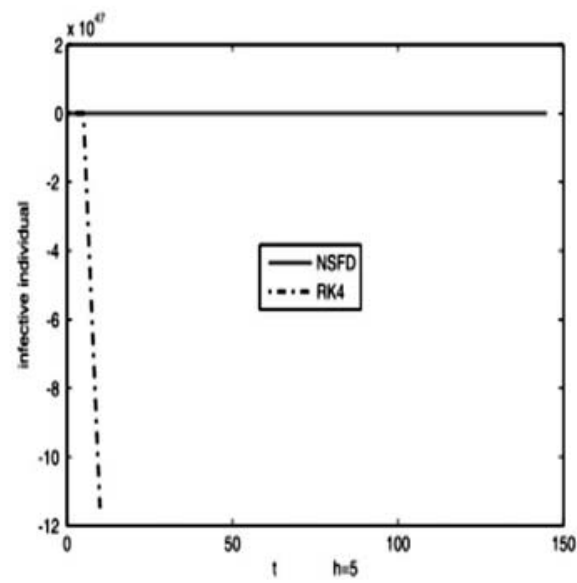

(b)

Fig. 7. Profile of infective individuals $I(t)$ using the 4-th order Runge-Kutta method (RK4) and the nonstandard numerical scheme (NSFD) (9) with the system 35.

Mickens, R. (2002). Nonstandard finite difference schemes for differential equations, Journal of Difference Equations and Applications 8(9): 823-847.

Mickens, R. (2005). Dynamic consistency: A fundamental principle for constructing nonstandard finite difference schemes for differential equations, Journal of Difference Equations and Applications 11(7): 645-653.

Moghadas, S., Alexander, M. and Corbett, B.D.and Gumel, A. (2003). A positivity preserving Mickens-type discretization of an epidemic model, Journal of Difference Equations and Applications 9(11): 1037-1051.

Moghadas, S. and Gumel, A. (2003). A mathematical study of a model for childhood diseases with non-permanent immunity, Journal of Computational and Applied Mathematics 157(2): 347-363.

Muroya, Y., Nakata, Y., Izzo, G. and Vecchio, A. (2011). Permanence and global stability of a class of discrete epidemic models, Nonlinear Analysis: Real World Applications 12(4): 2105-2117.

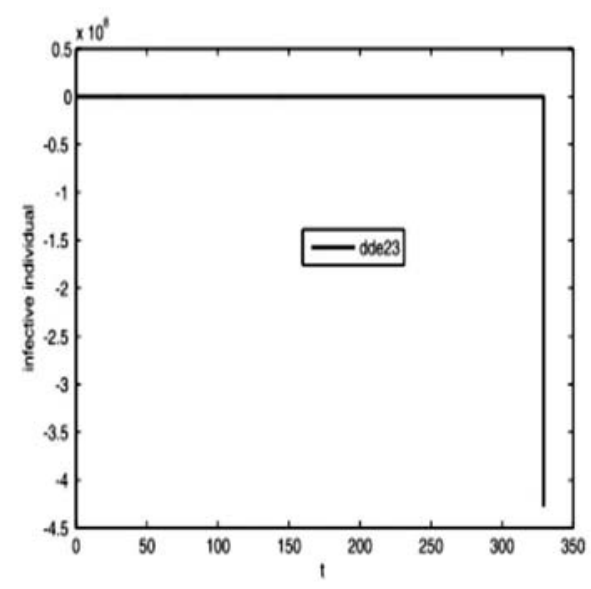

(a)

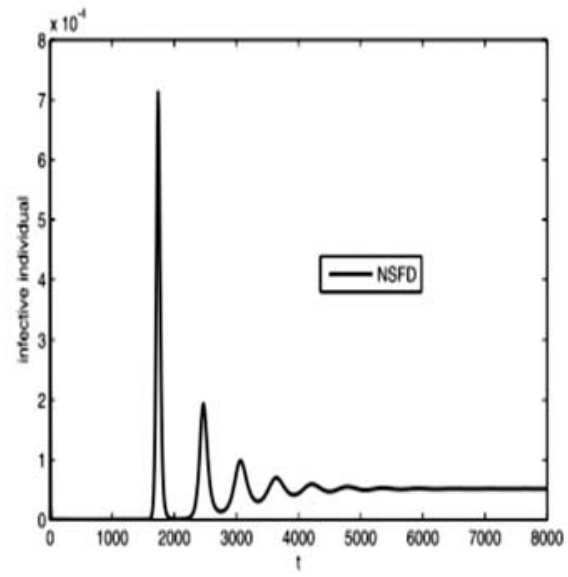

(b)

Fig. 8. Profile of an infected subpopulation $I(t)$ with the parameter values $a=0.002, p=1, d=0.002, b=500$, $\alpha=20$ and $\gamma=1\left(R_{0}>1\right)$ : using the Matlab mathematical package (a), under the numerical scheme (9) (b).

Obaid, H., Ouifki, R. and Patidar, K.C. (2013). An unconditionally stable nonstandard finite difference method applied to a mathematical model of HIV infection, International Journal of Applied Mathematics and Computer Science 23(2): 357-372, DOI: 10.2478/amcs-2013-0027.

Oran, E. and Boris, J. (1987). Numerical Simulation of Reactive Flow, Elsevier, New York, NY.

Parra, G.G., Arenas, A. and Charpentier, B.C. (2010). Combination of nonstandard schemes and Richardson's extrapolation to improve the numerical solution of population models, Mathematical and Computer Modelling 52(7): 1030-1036.

Potter, D. (1973). Computational Physics, Wiley-Interscience, New York, NY.

Sekiguchi, M. (2009). Permanence of some discrete epidemic models, International Journal of Biomathematics 2(4): 443-461. 
Sekiguchi, M. (2010). Permanence of a discrete SIRS epidemic model with time delays, Applied Mathematics Letters 23(10): 1280-1285.

Sekiguchi, M. and Ishiwata, E. (2010). Global dynamics of a discretized SIRS epidemic model with time delay, Journal of Mathematical Analysis and Applications 371(1): 195-202.

Stuart, A. and Humphries, A. (1996). Dynamic System and Numerical Analysis, Cambridge University Press, Cambridge.

Sundarapandian, V. (2003). An invariance principle for discrete-time nonlinear systems, Applied Mathematics Letters 16(1): 85-91.

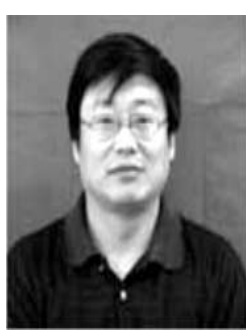

Xiaohua Ding received his Ph.D. degree in the Department of Mathematics, Harbin Institute of Technology, China. He became an associate professor in 1999, a full professor in 2004 and a doctoral tutor in 2007 at the same department. His research interests include numerical methods for delay differential equations, stochastic differential equations and impulsive differential equations, stability theory, dynamic systems for difference equations, and chaos synchronization.

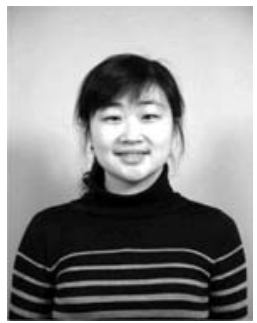

Deqiong Ding received her Ph.D. degree in the Department of Mathematics, Harbin Institute of Technology, China. She became a lecturer in 2011 at the same department. Her current research interests include numerical methods for delay differential equations, stability theory, and dynamic systems for difference equations.

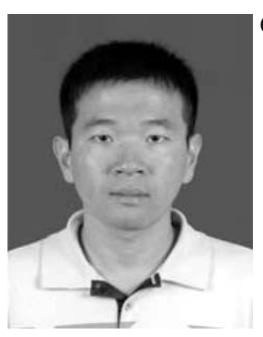

Qiang Ma received his Ph.D. degree at the Department of Mathematics, Harbin Institute of Technology, China, in 2013. He became a lecturer in 2013 at the same department. His current research interests include numerical methods for delay differential equations, stochastic differential equation and stability theory.

Received: 31 May 2013

Revised: 15 November 2013

Re-revised: 8 March 2014 